\title{
Dynamic Approach to Enhance Performance of Orthogonal Frequency Division Multiplexing (OFDM) In a Wireless Communication Network
}

\author{
James Agajo (M.Eng) ${ }^{1}$, Isaac O. Avazi Omeiza(Ph.D) ${ }^{2}$, Idigo Victor Eze(Ph.D) ${ }^{3}$,Okhaifoh Joseph(M.Eng.) ${ }^{4}$ \\ ${ }^{1}$ Dept. of Electrical and Electronic Engineering, , Federal Polytechnic, Auchi, Edo state, Nigeria \\ ${ }^{2}$ Dept. of Electrical and Electronics, University of Abuja, Nigeria \\ ${ }^{3}$ Dept. Electronics/Computer Engineering, Nnamdi Azikiwe University, Awka, Anambra state, Nigeria \\ ${ }^{4}$ Dept. of Electrical and Electronic, Federal University of Petroleum Resources Warri, Delta State Nigeria \\ Email: agajojul@yahoo.com
}

\begin{abstract}
In the mobile radio environment, signals are usually impaired by fading and multipath delay phenomenon. This work modeled and simulates OFDM in a wireless environment, it also illustrates adaptive modulation and coding over a dispersive multipath fading channel whereby simulation varies the result dynamically. Dynamic approach entails adopting probabilistic approach to determining channel allocation; First an OFDM network environment is modeled to get a clear picture of the OFDM concept. Next disturbances such as noise are deliberately introduced into systems that are both OFDM modulated and non-OFDM modulated to see how the system reacts. This enables comparison of the effect of noise on OFDM signals and nonOFDM modulated signals. Finally efforts are made using digital encoding schemes such as QAM and DPSK to reduce the effects of such disturbances on the transmitted signals. In the mobile radio environment, signals are usually impaired by fading and multipath delay phenomenon. In such channels, severe fading of the signal amplitude and inter-symbol-interference (ISI) due to the frequency electivity of the channel cause an unacceptable degradation of error performance. Orthogonal frequency division multiplexing (OFDM) is an efficient scheme to mitigate the effect of multipath channel.
\end{abstract}

Keywords- OFDM, Inter-Carrier Interference, IFFT, multipath,Signal.

\section{INTRODUCTION}

Mobile radio communication systems are increasingly demanded to provide a variety of high- quality services to mobile users. To meet this demand, modern. mobile radio transceiver system must be able to support high capacity, variable bit rate information transmission and high bandwidth efficiency. In the mobile radio environment, signals are usually impaired by fading and multipath delay phenomenon. In such channels, severe fading of the signal amplitude and intersymbol-interference (ISI) due to the frequency selectivity of the channel cause an unacceptable degradation of error performance. Orthogonal frequency division multiplexing (OFDM) is an efficient scheme to mitigate the effect of multipath channel. Since it eliminates ISI by inserting guard interval (GI) longer than the delay spread of the channel [1], [2]. Therefore, OFDM is generally known as an effective technique for high data rate services. Moreover, OFDM has been chosen for several broadband WLAN standards like IEEE802.11a, IEEE802.11g, and European HIPERLAN/2, and terrestrial digital audio broadcasting (DAB) and digital video broadcasting (DVB) was also proposed for broadband wireless multiple access systems such as IEEE802.16 wireless MAN standard and interactive DVB-T [3], In OFDM systems, the pilot signal averaging channel estimation is generally used to identify the channel state information (CSI) [5]. In this case, large pilot symbols are required to obtain an accurate CSI. As a result, the total transmission rate is degraded due to transmission of large pilot symbols. Recently, carrier interferometry (CI) has been proposed to identify the CSI of multiple-input multiple-output (MIMO). However, the CI used only one phase shifted pilot signal to distinguish all the CSI for the combination of transmitter and receiver antenna elements.[3,4]

In this case, without noise whitening, each detected channel impulse response is affected by noise [6]. Therefore, the pilot signal averaging process is necessary for improving the accuracy of CSI [7]. To reduce this problem, time, frequency interferometry (TFI) for OFDM has been proposed. [8] - [10].

The main problem with reception of radio signals is fading caused by multipath propagation. There are also inter-symbol interference (ISI), shadowing etc. This makes link quality vary. As a result of the multipath propagation, there are many reflected signals, which arrive at the receiver at different times. Some of these reflections can be avoided by using a directional antenna, but it is impossible to use them for a mobile user. A solution could be usage of antenna arrays, but this technology is still being developed.

This is why this research and development of the OFDM have received considerable attention and have made a great deal of progress in all parts of the world. OFDM is a wideband modulation scheme that is specifically able to cope with the problems of the multipath reception. This is achieved by transmitting many narrowband overlapping digital signals in parallel, inside one wide band. [5] 


\section{A. Objective of Project}

The aim of this project is to simulate the physical layer of an OFDM system. It investigates the OFDM system as a whole and provides a simple working model on which subsequent research can be built. Hence the successful completion of this work shall involve;

1) Practical description of the OFDM system.

2) Algorithm development based on mathematical analysis of the OFDM scheme.

3) Modeling of the algorithm and a software test based on the MATLAB/Simulink environment.

Thus a typical OFDM system modeling the data source, the transmitter, the air channel and the receiver side of the system is simulated.

This project is intended to model and simulate an OFDM network environment. A simple data source is provided to serve as the input; likewise the transmitter, channel and receiver are modeled using appropriate block-sets in the Simulink. A simple representation of the OFDM system is modeled, though with little deviation from the real implementation. But all efforts had been taken in this work to reduce the effects of such deviations.[6]

\section{B. OVERVIEW}

Orthogonal Frequency Division is where the spacing between carriers is equal to the speed (bit rate) of the message. In earlier multiplexing literature, a multiplexer was primarily used to allow many users to share a communications medium like a phone trunk between two telephone central offices. In OFDM, it is typical to assign all carriers to a single user; hence multiplexing is not used with its generic meaning.

Orthogonal frequency division multiplexing is then the concept of typically establishing a communications link using a multitude of carriers each carrying an amount of information identical to the separation between the carriers. In comparing OFDM and single carrier communication systems (SCCM), the total speed in bits per second is the same for both, $1 \mathrm{Mbit} / \mathrm{sec}$ (Mbps) in this example. For single carrier systems, there is one carrier frequency, and the $1 \mathrm{Mbps}$ message is modulated on this carrier, resulting in a $1 \mathrm{MHz}$ bandwidth spread on both sides of the carrier. For OFDM, the 1,000,000 bps message is split into 10 separate messages of $100,000 \mathrm{bit} / \mathrm{sec}$ each, with a $100 \mathrm{KHz}$ bandwidth spread on both sides of the carrier.[7]

To illustrate how frequencies change with time, we can use the analogy of the sounds of an orchestra or band. One carrier wave is analogous to one instrument playing one note, whereas many carriers is analogous to many instruments playing at once. Single carrier systems using a high speed message is analogous to a drum roll where the sticks are moving fast.

A more detailed understanding of Orthogonal arises when we observe that the bandwidth of a modulated carrier has a so called sinc shape $(\sin x / x)$ with nulls spaced by the bit rate. In OFDM, the carriers are spaced at the bit rate, so that the carriers fit in the nulls of the other carriers.

\section{CHOICE OF APPROACH}

The bottom-up design approach is chosen for this work because of its concise form and ease of explanation.

\section{A. Modeling the OFDM system}

For the simulation, the Signal Processing and the Communication Block-sets are used. The OFDM network can be divided into three parts i.e. the transmitter, receiver and channel. A data source is also provided which supplies the signal to be transmitted in the network. Thereafter, the bit error rate can be calculated by comparing the original signal at the input of the transmitter and the signal at the output of the receiver.

\section{Transmitter}

Convolutional encoder. In order to decrease the error rate of the system, a simple convolution encoder of rate $1 / 2$ is used as channel coding.

\section{Interleaver.}

The interleaver rearranges input data such that consecutive data are split among different blocks. This is done to avoid bursts of errors. An interleaver is presented as a matrix. The stream of bits fills the matrix row by row. Then, the bits leave the matrix column by column. The depth of interleaver can be adjusted.

\section{Modulation.}

- A modulator transforms a set of bits into a complex number corresponding to a signal constellation. The modulation order depends on the subcarrier.

- Bits flow through an interleaver with high SNR will be assigned more bits than a subchannel with low SNR. Modulations implemented here are QPSK, 16QAM and 64QAM.

- Symmetrical IFFT. Data are transformed into timedomain using IFFT. The total number of subcarriers translates into the number of points of the IFFT/FFT. A mirror operation is performed before IFFT in order to get real symbols as output

- Cyclic Prefix (CP). To preserve the orthogonality property over the duration of the useful part of signal, a cyclic prefix is then added. The cyclic prefix is a copy of the last elements of the frame.

- D/A. Convert digital symbols to analog signals. This operation is done using the AIC codec inside the DSP.

- Channel[8]

- The channel must have the same characteristics as the pair of twisted wires found in the telephone network. In order to achieve this, we use a telephone line emulation hardware. Also, we have the possibility to use the adjustable filter ZePo and the noise generator. This can be very useful to test the system performance. 


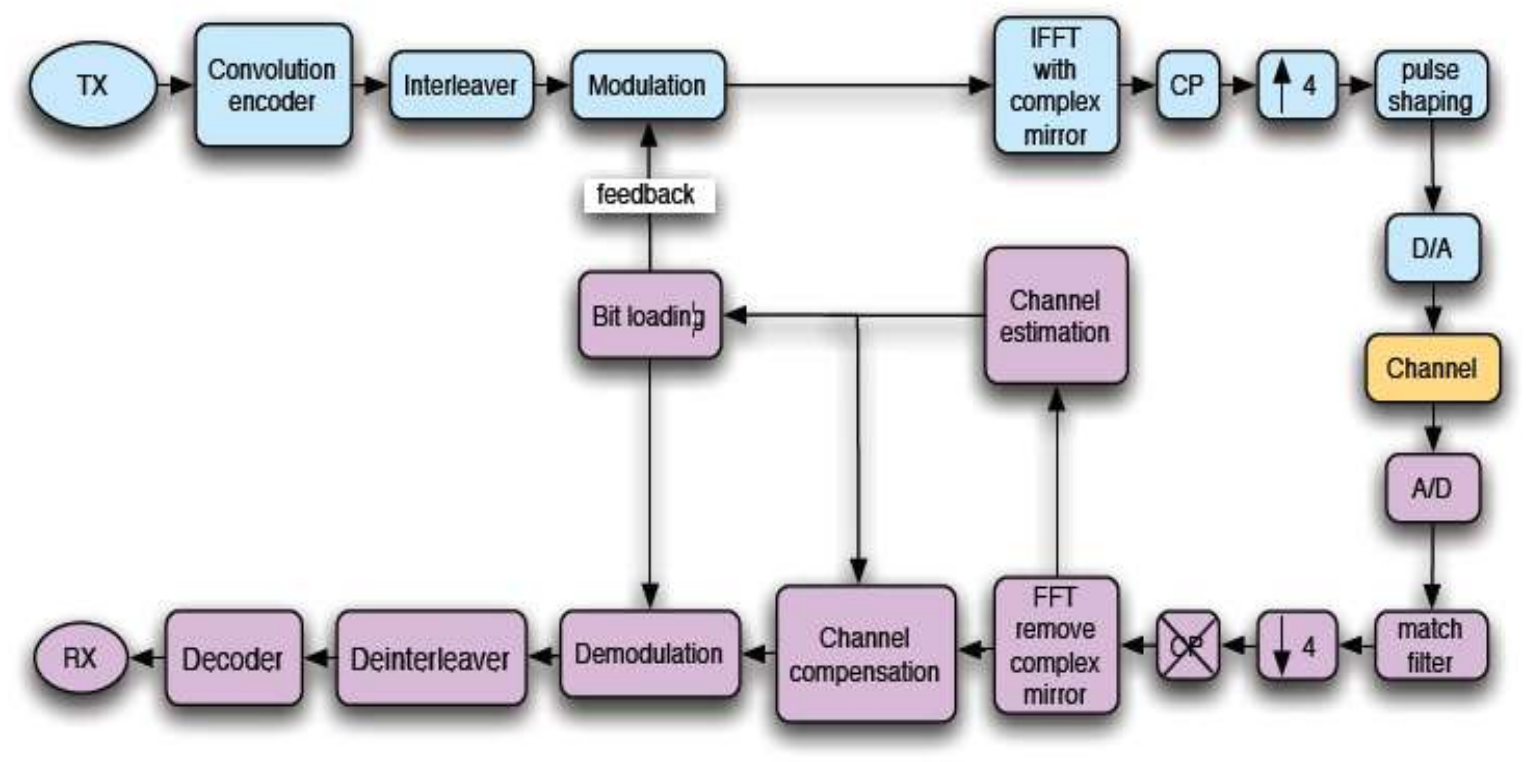

Fig. 2.0 OFDM system

\section{B. Receiver}

- A/D. Convert analog signals to digital symbols for processing.

- Synchronization. Due to the clock difference between transmitter and receiver, a synchronization algorithm is needed to find the first sample in the OFDM frame.

- Remove cyclic prefix. This block simply removes the cyclic prefix added in the transmitter.

- Symmetrical FFT. Data are transformed back to frequency-domain using FFT. Then the

- complex conjugate mirror added in the transmitter is removed.

- Channel estimation. The estimation is achieved by pilot frames.

- Channel compensation. The channel estimation is used to compensate for channel distortion.

- Bit loading. The receiver computes the bit allocation and send it to the transmitter.[9]

- Demodulation. Symbols are transformed back to bits. The inverse of the estimated channel response is used to compensate the channel gain.

- Deinterleaver (Interleaving inverse operation). The stream of bits fills the matrix column by column. Then, the bits leave the matrix row by row.

- Convolution decoder. The decoder performs the Viterbi decoding algorithm to generate transmitted bits from the coded bits.
We assume that a propagation channel consists of $L$ discrete paths with different time delays. The impulse response $h(\tau, t)$ is represented as

$$
h(\tau, t)=\sum_{l=0} h l(\tau) \delta(\tau-t)
$$

where $h l$ and $\tau l$ are complex channel gain and the time delay of $l$ th propagation path, respectively,

The channel transfer function $H(f, t)$ is the Fourier transform of $h(\tau, t)$ and is given by

$$
\begin{gathered}
H(f, t)=\int_{0}^{\infty} h(\tau, t) \exp (-j 2 \pi f t) d t \cdots . . \\
=\sum_{l=0}^{L-1} h l \exp (-j 2 \pi f \tau l) \\
\mathrm{g}(\mathrm{t}) \quad \ldots . \\
\quad 1 \text { for }-\mathrm{Tg}<\mathrm{t}<\mathrm{Ts} \\
0 \text { otherwise }
\end{gathered}
$$

The guard interval $I g$ is inserted in order to eliminate the ISI due to the multi-path fading, and hence, we have

$$
. T=T s+T g
$$

In OFDM systems, $T g$ is generally considered as $T s / 4$ or $T s / 5$. Thus, we assume $T g=T s / 4$ in this paper. In (3), $g(t)$ is the transmission pulse which gives $\mathrm{g}(\mathrm{t})$ 


\section{The Simulation Process}

The simulation process is carried out in stages using different digital modulators, and considering different effects of the wireless interface. Hence, the wireless channel effects are varied, as well as the type of digital modulators used for the OFDM modulation. The effect of additive white Gaussian noise (AWGN), is considered on a signal which is QAM modulated. Finally, the combined effect of Phase noise and AWGN on QAM modulated OFDM signal is modeled and simulated. This enables comparison of the effect of noise on OFDM signals using QAM modulation.[11]

\section{Mathematical analysis of OFDM system.}

This system compares the Error Rates of an OFDM modulated signal with that of a non-OFDM modulated signal. The error rates of an OFDM modulated are expected to be lower than those of non-OFDM

$$
S_{c}(t)=A_{c}(t) e^{j\left[\omega_{c} t+\phi_{c}(t)\right]}
$$

Where

$\omega_{n}=\omega_{0}+n \Delta \omega$

This is of course a continuous signal. If we consider the waveforms of each component of the signal over one symbol period, then the variables $\mathrm{Ac}(\mathrm{t})$ and $\mathrm{fc}(\mathrm{t})$ modulated signals which be written as equation 2.8 .

\section{OFDM spectrum}
(a) A single Sub-channel
(b) Five carriers

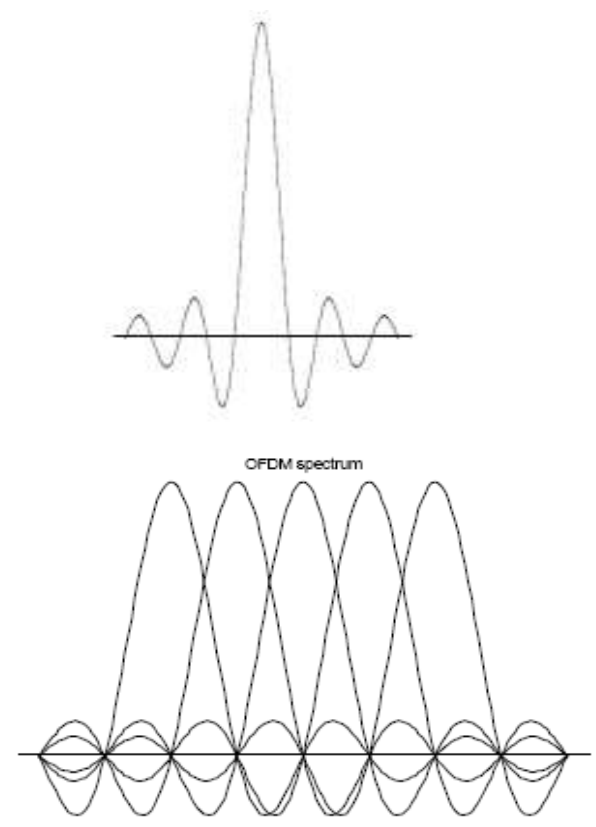

Fig 2.1 Examples of OFDM spectrum
Mathematically, each carrier can be described as a complex wave; [3] take on fixed values, which depend on the frequency of that particular carrier, and so can be rewritten as:

$$
\begin{aligned}
& \phi_{n}(t) \Rightarrow \phi_{n} \\
& A_{n}(t) \Rightarrow A_{n .}(2.8)
\end{aligned}
$$

If the signal is sampled using a sampling frequency of $1 / T$, then the resulting signal is represented by:

$$
S_{s}(k T)=\frac{1}{N} \sum_{n=0}^{N-1} A_{n} e^{j\left[\left(\omega_{0}+n \Delta \omega\right) k T+\phi_{n}\right]}
$$

At this point, we have restricted the time over which we analyze the signal to $\mathrm{N}$ samples. It is convenient to sample over the period of one data symbol. Thus we have a relationship

$$
\tau=N T
$$

If we now simplify eqn. (2.9), without a loss of generality by letting $\mathrm{w} 0=0$, then the signal becomes:

$$
S_{s}(k T)=\frac{1}{N} \sum_{n=0}^{N-1} A_{n} e^{j \phi_{n}} e^{j(n \Delta \omega) k T}
$$

Now Eq. (2.10) can be compared with the general form of the inverse Fourier transform:

$$
g(k T)=\frac{1}{N} \sum_{n=0}^{N-1} G\left(\frac{n}{N T}\right) e^{j 2 \pi n k / N}
$$

In eq. (2.10) and (2.11), the function $s(k T)$ is no more than a definition of the signal in the sampled frequency Domain, and $A_{n} e^{j \phi_{n}}$ is the time domain representation. Eqns. (2.10) and (2.11) are equivalent if:

$$
\Delta f=\frac{\Delta \omega}{2 \pi}=\frac{1}{N T}=\frac{1}{\tau}
$$

\section{E. Factors influencing the control system}

\section{1) Signal-To- noise Ratio}

AWGN is additive, which means that the noise signal adds to the existing signal, resulting in a distorted version of the original signal.

It is possible to determine the quality of a digitally modulated signal influenced by AWGN using the probability density function and the standard deviation signal. The signal 
to- noise ratio is defined as the ratio [4] of the power of the signal to the noise power.

$S N R=\left(S_{\text {power }} / N_{\text {power }}\right)=\left(S_{r m s}\left(t, f_{c}\right) / n_{r m s}\left(t, f_{c}\right)\right)^{2}$

Or in decibel;

\section{$S N R_{d B}=10 \log _{10}(S N R)$}

\section{F. Probability of Error in QPSK modulation}

Because of the randomness of AWGN, it is impossible to predict the exact locations of incorrectly decoded bits, it is however possible to theoretically predict the amount of incorrectly decoded bits in the long run, and from that calculate error probabilities like the symbol- error rates and bit- error rates.

$$
P\left(U_{00} / S_{10}\right)=P(-<x<0)=\int_{2 \pi}^{01} \ell^{-(x-(4.92)) /(2 \sqrt{2} d x)}
$$

QPSK encodes two data bits into a sinusoidal carrier wave by altering the sinusoidal carrier wave's phase. The probability that a QPSK decoder will incorrectly decode a symbol $U_{00}$ given that the correct transmitted symbol was $S_{10}$ is given as [5].

\section{$\mathrm{SNR}_{\mathrm{QPSK}-\mathrm{Db}}=$}

$=\left[10 \log _{10} \text { QPSKsignalpower } / \text { NoisePower }\right]_{+} 10 \log _{10}(52)$

TABLE 2.1 : THE OFDM SYMBOL DECODING PROCESS

\begin{tabular}{|l|l|l|}
\hline SNR & BER & SER \\
\hline 2 & .11 & .05 \\
\hline 4 & .33 & .28 \\
\hline 6 & .55 & .45 \\
\hline 8 & .69 & .63 \\
\hline 10 & .75 & .70 \\
\hline 12 & .80 & .74 \\
\hline 14 & .85 & .76 \\
\hline 16 & .89 & .79 \\
\hline 18 & .91 & .80 \\
\hline
\end{tabular}

Unfortunately, this function is not directly solvable and look up tables are used to determine the results.
There exists a function, though that is closely related to $\mathrm{p}$, The Q- function [5]

The total symbol error rate of a QPSK decoder can finally be calculated as the average symbol error probability of $p\left(U_{00} / S_{10}\right), p\left(U_{01} / S_{01}\right), p\left(U_{10} / S_{10}\right)$

and $p\left(U_{11} / S_{10}\right)$

The SER is given as

$S E R=\lfloor 2 Q(A / \sqrt{2})\rfloor$

The Bit- Error- Rate of a QPSK decoder is given as:

$B E R=\lfloor Q(A / \sqrt{2})\rfloor$ have:

Expressing the SER and BER as a function of SNR, we

$$
\begin{aligned}
& S E R=\lfloor 2 Q(\sqrt{S N R})\rfloor . \\
& B E R=\lfloor Q(\sqrt{S N R})\rfloor .
\end{aligned}
$$

From the above relationships of eqns (2.19) and (2.20), the plot for the BER \& SER of a QPSK modulated signal is as

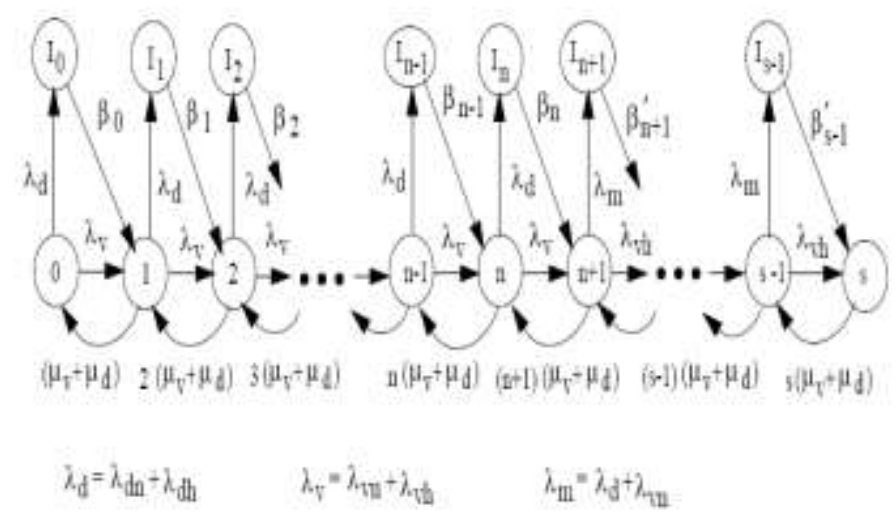

shown below

Figure 2.2: shows the state transition diagram for the model of a cell operating under the proposed algorithm.

SNR $\operatorname{QPSK}_{-d B}=\left[10 \log _{10}\right.$ QPSKsignalpower/NoisePower $]$ - -2.21

Since IEEE 802.11a OFDM signal has $N_{s t}=52 \ldots 2.22$

QPSK sub - carriers, the signal has 52 times more power 


$$
\text { : } S N R_{d B}-O F D M=S N R_{d B}-Q P S K+17.6 d B(4.99 \mathrm{~d}) . .
$$

\section{G. STATE TRANSITION DIAGRAM}

It is a very useful pictorial representation that clearly shows the protocol (rule) operation.

Thus, it's a tool to design the electronics that implements the protocol and troubleshoots communication problems. In a state diagram, all possible activity states of the system are shown in nodes. At each state node, the system must respond to some event occurring and then proceed to the appropriate next state. [9]

\section{H. Probabilitic Channel Allocation in OFDM}

For a cell having $\mathrm{S}$ channels, the model has $2 \mathrm{~s}+1$ states, namely $0,1,2,3, \mathrm{~S}, \mathrm{I}_{\mathrm{O}}, \mathrm{I}, \ldots \ldots \ldots \mathrm{I}_{\mathrm{S}-1}$.

A cell is defined as cold cell if it is in state $K$, for $0 \leq K \leq n$, whereas a cell is called hot cell if it is not a state greater than $n$. If a data call with rate $\lambda d$ (respectively, a voice call with rate $\lambda v$ ) arrives in a cold cell with state $K$, then the cell enters state $\mathrm{I}_{\mathrm{K}}$ (respectively, state $\mathrm{K}+1$ ). On the other hand, if a data call with rate $\lambda \mathrm{m}$ or a new voice call with rate $\lambda \mathrm{vn}$ arrives in a hot cell in state $\mathrm{j}$ for $\mathrm{j}>\mathrm{n}$, then the cell enters state $\mathrm{Ij}$. A handoff voice call is always assigned a whole channel. However, a new voice is assigned a whole channel if the cell is a cold cell.

Let $\mathrm{Pj}$ denote the steady state probability that the process is in state $\mathrm{j}$, for $\mathrm{j}=0 ; 1 ; 2$; _ _ $; \mathrm{S}$. Assuming that all channel holding times are exponentially distributed.

For $\mathrm{j}=0$ (i.e., states 0 and $\mathrm{I}_{0}$ ):

$(\lambda \mathrm{d}+\lambda \mathrm{v}) \mathrm{P}_{0}=\mathrm{P}_{1}(\mu \mathrm{d}+\mu \mathrm{v})$

$\Lambda \mathrm{d} \mathrm{P}_{0}=\mathrm{P} \mathrm{I}_{0} \beta_{0}$.

It follows from (1) that

$\mathrm{P}_{1}=\frac{\lambda \mathrm{d}+\lambda \mathrm{v} \mathrm{P} 0=}{\mu \mathrm{v}+\mu \mathrm{d}} \mathrm{P}_{0 \ldots \ldots \ldots \ldots \ldots \ldots \ldots \ldots . .(2.26)}$

For $\mathrm{j}=1 ; 2 ; \ldots \ldots . \mathrm{n}$

$(\lambda d+\lambda v) P j+j(\mu v+\mu d) P j=\lambda v P_{j-1}+(j+1)(\mu v+\mu d) P_{j+1}+$

$\mathrm{PI}_{\mathrm{j}-1} \beta_{\mathrm{j}-1}$

$\lambda \mathrm{dPj}=\operatorname{PIj} \beta \mathrm{j}$

Eq. (1.5) implies that $\lambda d \mathrm{P}_{\mathrm{j}-1}$ can be substituted for $\mathrm{PI}_{\mathrm{j}-1} \beta_{\mathrm{j}-1}$ in (4.4). Hence, Eq. (4.4) can be rewritten as

$(\lambda d+\lambda v) P_{j}+j(\mu v+\mu d) P j=\lambda v P_{j-1}+(j+1)(\mu v+\mu d) P_{j+1}+$ $\lambda d P_{j-1}=(\lambda d+\lambda v) P_{j-1}+(j+1)(\mu d+\mu v) P_{j+1}$

By solving (1.6) recursively by letting $\mathrm{j}=1 ; 2 ; 3$ and $n-1$ in order to obtain

$\mathrm{P}_{2}=1 / 2 ! \rho^{2} \mathrm{P}_{0}$

$\mathrm{P}_{3}=1 / 3 ! \rho^{3} \mathrm{P}_{0}$

$\mathrm{P}_{4}=1 / 4 ! \rho^{4} \mathrm{P}_{0}$
$\mathrm{P}_{5}=1 / 5 ! \rho^{5} \mathrm{P}_{0}$ and

$\mathrm{P}_{\mathrm{n}}=1 / \mathrm{n} ! \rho^{\mathrm{n}} \mathrm{P}_{0}$ respectively.

Therefore, for $0 \leq \mathrm{j} \leq \mathrm{n}$, we obtain

$P j=1 / j ! \rho^{j} P_{0}$

For $\mathrm{j}=\mathrm{n}+1, \mathrm{n}+2, \ldots \ldots \ldots \ldots . \mathrm{S}-1$, we have the following balance equation in the equilibrium case

$(\lambda \mathrm{m}+\lambda v h) \mathrm{P}_{\mathrm{j}}+\mathrm{j}(\mu \mathrm{v}+\mu \mathrm{d}) \mathrm{Pj}=\lambda v \mathrm{vPP}_{\mathrm{j}-1}+(\mathrm{j}+1)(\mu \mathrm{v}+\mu \mathrm{d}) \mathrm{P}_{\mathrm{j}+1}+$ $\lambda \mathrm{mPI}_{\mathrm{j}-1}(\lambda \mathrm{d}+\lambda \mathrm{v}) \mathrm{P}_{\mathrm{j}}+\mathrm{j}(\mu \mathrm{d}+\mu \mathrm{v}) \mathrm{Pj}=(\lambda \mathrm{d}+\lambda \mathrm{v}) \mathrm{P}_{\mathrm{j}-1}+$ $(\mathrm{j}+1)(\mu \mathrm{d}+\mu \mathrm{v}) \mathrm{P}_{(\mathrm{j}+1)}$

Where $\lambda \mathrm{d}+\lambda \mathrm{v}=\lambda \mathrm{m}+\lambda \mathrm{vh}$

Note that Equation (10) is the same as Equation (1.6) therefore Equ. (4.7) also holds for $j=n+1, n+2, \ldots \ldots . . S-1$.

For $\mathrm{j}=\mathrm{S}$

$\mathrm{S}(\mu \mathrm{v}+\mu \mathrm{d}) \mathrm{P}_{\mathrm{s}}=\lambda v h \mathrm{P}_{\mathrm{s}-1}+\mathrm{PI}_{\mathrm{s}-1} \beta^{\prime}{ }_{\mathrm{j}-1}=\lambda v h \mathrm{PI}_{\mathrm{s}-1}=(\lambda v h+\lambda \mathrm{m}) \mathrm{P}_{\mathrm{s}-1}$ $=(\lambda \mathrm{d}+\lambda \mathrm{v}) \mathrm{P}_{\mathrm{s}-1} \ldots \ldots(2.31)$

$\mathrm{P}_{\mathrm{s}}=1 / \mathrm{S} ! \rho^{\mathrm{s}} \mathrm{P}_{0}$.

Thus for $0 \leq \mathrm{J} \leq \mathrm{S}$, the steady state probability, $\mathrm{P}_{\mathrm{j}}$ is

$\mathrm{P}_{\mathrm{j}}=1 / \mathrm{J} ! \rho^{\mathrm{j}} \mathrm{P}_{0}$

The $\mathrm{P}_{0}$ for handoff dropping probability, $\mathrm{P}_{0 \mathrm{~d}}$, and new call blocking probability, $\mathrm{P}_{0 \mathrm{~b}}$ are equal to the same steady-state probability $\mathrm{P}_{0 \mathrm{~s}}$, that is,

$\mathrm{P}_{0}$ for $\mathrm{P}_{\mathrm{d}}=\mathrm{P}_{\mathrm{b}}=\mathrm{P}_{\mathrm{s}}$

If a handoff calls requests a free packet slots of a channel and is available. Then based on the algorithm proposed

$\mathrm{P}_{\mathrm{d}}=1 / 3 \mathrm{j} ! \rho^{\mathrm{j}} \mathrm{P}_{0}$

When a new call is assigned a channel, the call is also assigned a channel holding time, which is generated by an exponential distribution function with a mean value of 15 time slots. Call arrival is modeled with Markov Chain as a Poisson process with different mean arrival rates, and the call duration is exponentially distributed with a mean value of 15 time slots. The traffic is characterized by the arrival rate of new calls and by the transition probabilities of handoff calls. It is assumed that base station has a buffer with a substantially large buffer capacity to avoid significant packet loss.

\section{SYSTEM IMPLEMENTATION}

\section{A. Software Subsystem Implementation}

The OFDM system was modeled and simulated using MATLAB \& Simulink to allow various parameters of the system to be varied and tested, including those established by the standard as shown in fig 5.1 the simulation includes all the stages for transmitter, channel and receiver, according to the standard. Because of the MATLAB sampling time, the transmission was implemented in baseband to avoid long periods of simulation. Considering additive white gaussian noise (AWGN) and multipath path Rayleigh fading effect, a good approximation to the real performance can be observed, 
over all in the degradation of the BER. At the transmitter, OFDM signals are generated by Bernoulli Binary and mapped by one of the modulation techniques. Then by using a Sequence Generation, The transmitter section converts digital data to be transmitted, into a mapping of sub carrier amplitude and phase. It then transforms this spectral representation of the data into the time domain using an Inverse Fast Fourier Transform (IFFT). The OFDM symbol is equal to the length of the IFFT size used (which is 1024) to generate the signal and it has an integer number of cycles. The Cyclic Prefix and Multiple Parameters were added before the signal conversion from Parallel to Serial mode. The addition of a guard period to the start of each symbol makes further improvement to the effect of ISI on an OFDM signal. For generation an OFDM signal, all the model variables parameters were setting in suitable values in order to have smooth generated signal to be transmitted. The channel simulation will allow for us to examine the effects of noise and multipath on the OFDM scheme. By adding small amount of random data of AWGN to the transmitted signal, noise can be simulated. Generation of random data at a bit rate that varies during the simulation. The varying data rate is accomplished by enabling a source block periodically for a duration that depends on the desired data rate.

The result above denotes the effect of noise (AWGN) on a non-OFDM modulated signal. This result is to be compared with the result of fig (4.3) so as to draw a comparison between the effect of noise on a non-OFDM modulated data signals and that of an OFDM modulated signal.

It is expected that OFDM performs better in noisy and disturbed environment than any other modulation technique compared with it here. It is also expected that the Bit Error Rate (BER) and Symbol Error Rate (SER) of OFDM modulated signals is always less than that of a non-OFDM modulated signals. The theoretical symbol error probability of PSK is Where erfc is the complementary error function, $E_{S} / N_{O}$ is the ratio of energy in a symbol to noise power spectral density, and $\mathrm{M}$ is the number of symbols.

$$
P_{E}(M)=\operatorname{erfc}\left(\sqrt{\frac{E_{\mu}}{N_{\circ}}} \sin \left(\frac{\pi}{M}\right)\right)
$$

To determine the bit error probability, the symbol error probability, PE, needs to be converted to its bit error equivalent. There is no general formula for the symbol to bit error conversion. Upper and lower limits are nevertheless easy to establish. The actual bit error probability, $\mathrm{Pb}$, can be shown to be bounded by

$$
\frac{P_{E}(M)}{\log _{2} M} \leq P_{b} \leq \frac{M / 2}{M-1} P_{E}(M) \ldots
$$

The lower limit corresponds to the case where the symbols have undergone Gray coding. The upper limit corresponds to the case of pure binary coding. Because increasing the value of $\mathrm{Eb} / \mathrm{No}$ lowers the number of errors produced, the length of each simulation must be increased to ensure that the statistics of the errors remain stable

Using the sim command to run a Simulink simulation from the MATLAB Command Window, the following code generates data for symbol error rate and bit error rate curves. It considers Eb/No values in the range $0 \mathrm{~dB}$ to $12 \mathrm{~dB}$, in steps of $2 \mathrm{~dB}$.

\section{Simulation Result}

The importance of modulation using (OFDM) can be seen in the above simulations, since the un-modulated signal always performs poorer than the modulated signal. Hence, modulation makes a signal more conducive for transmission over the transmission medium (in this case, the wireless channel). It is also observed that appropriate choice of modulation techniques could either increase or decrease the error rates of the signals. Hence, a DPSK-modulated OFDM signal fig (4.6) is much more conducive for transmission over the wireless channel than any other type of modulation tested. The effect of the Additive White Gaussian Noise (AWGN) is observed by modeling a signal passing through a noisy channel without any form of modulation. Afterwards, OFDM modulated signals (using digital modulators such as QAM) are passed through the same channel. The error rate is then compared.

A matlab file (see Appendix A) is written to vary the signal-to-noise ratio (SNR) and plot the graph of the Bit Error Rate (BER). The BER of the un-modulated signal is found to be constant at 0.4904 . The effect of a noisy channel on a QAM signal is modeled as shown in fig 4.4. It is observed that an unmodulated signal has a BER of about 50\%, whereas OFDM modulation reduces the BER significantly. It was also observed that the DPSK-modulated OFDM signal reduces the BER significantly, graph on Fig 4.7 shows a probabilistic approach on the Comparison of the outage probability with the signal to interference ratio of $\mathrm{CCI}$, Appendiv 1 and Appendix 2 represent a classical representation of how OFDM and QPSK simulation. 


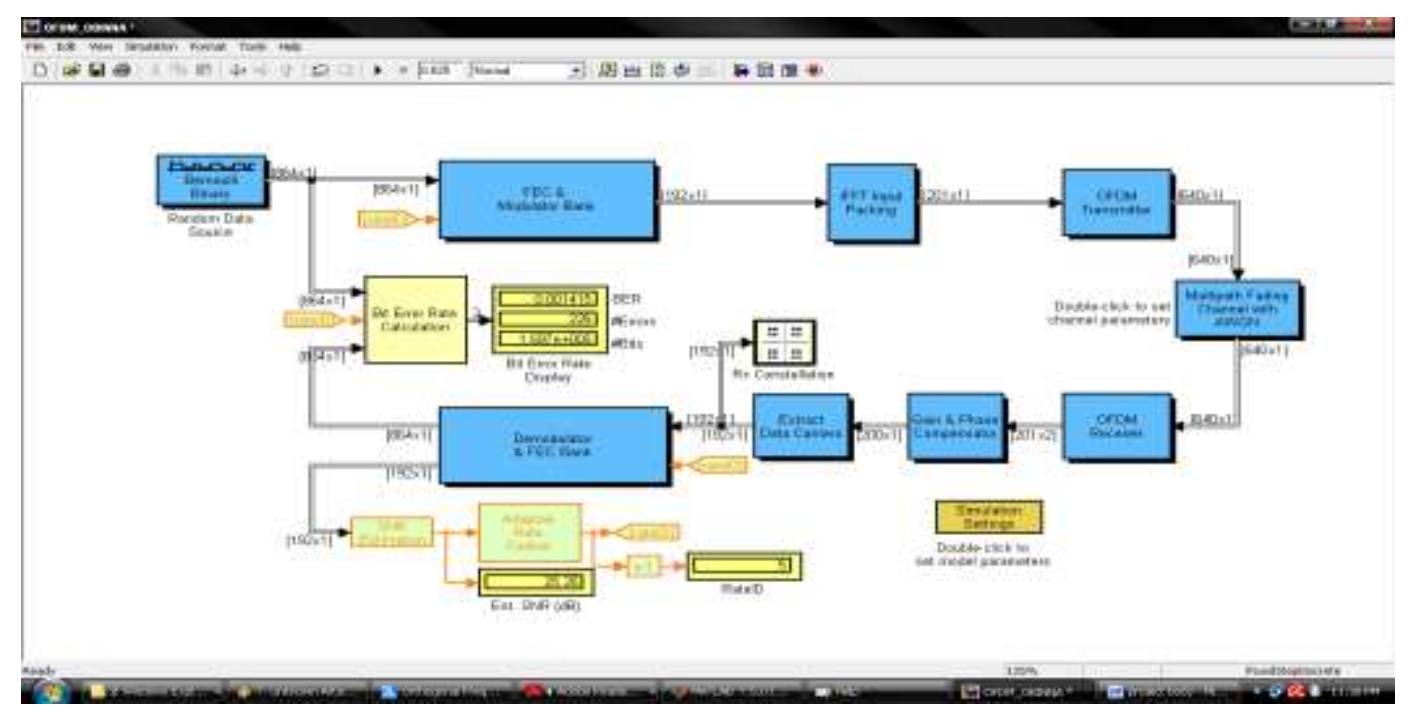

Fig 4.1 Modeling an OFDM network environment

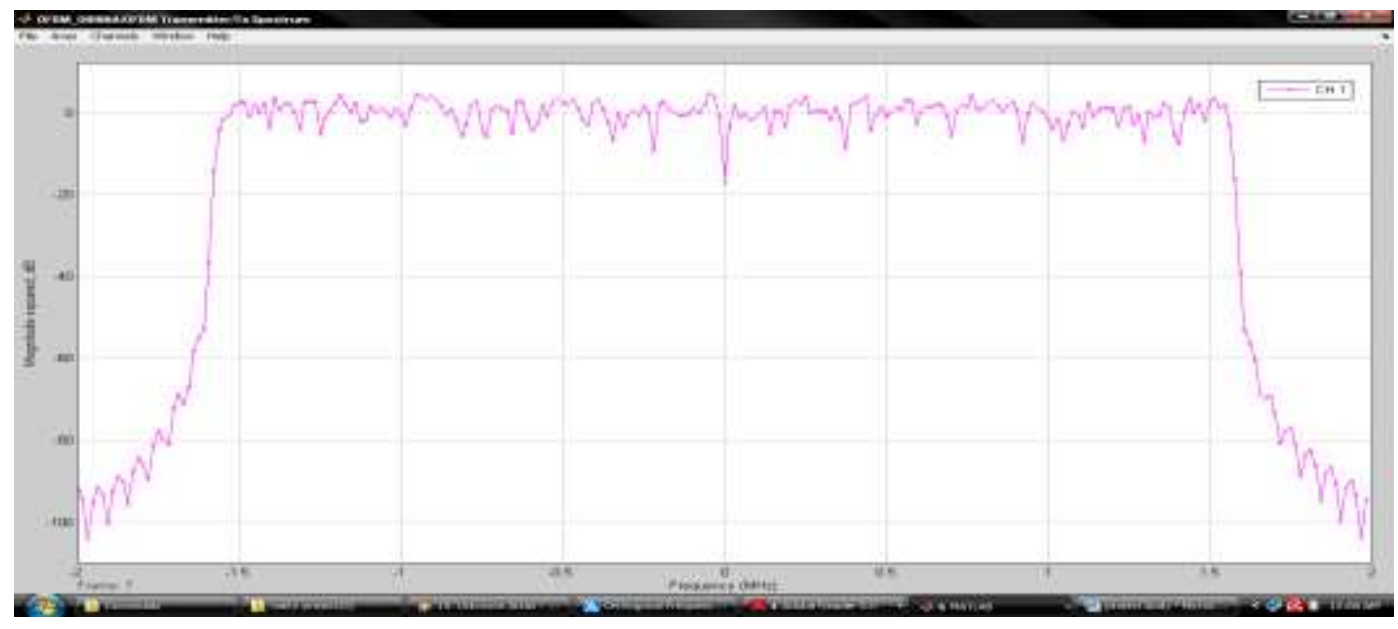

Fig 4.2 Graph of Transmission spectrum

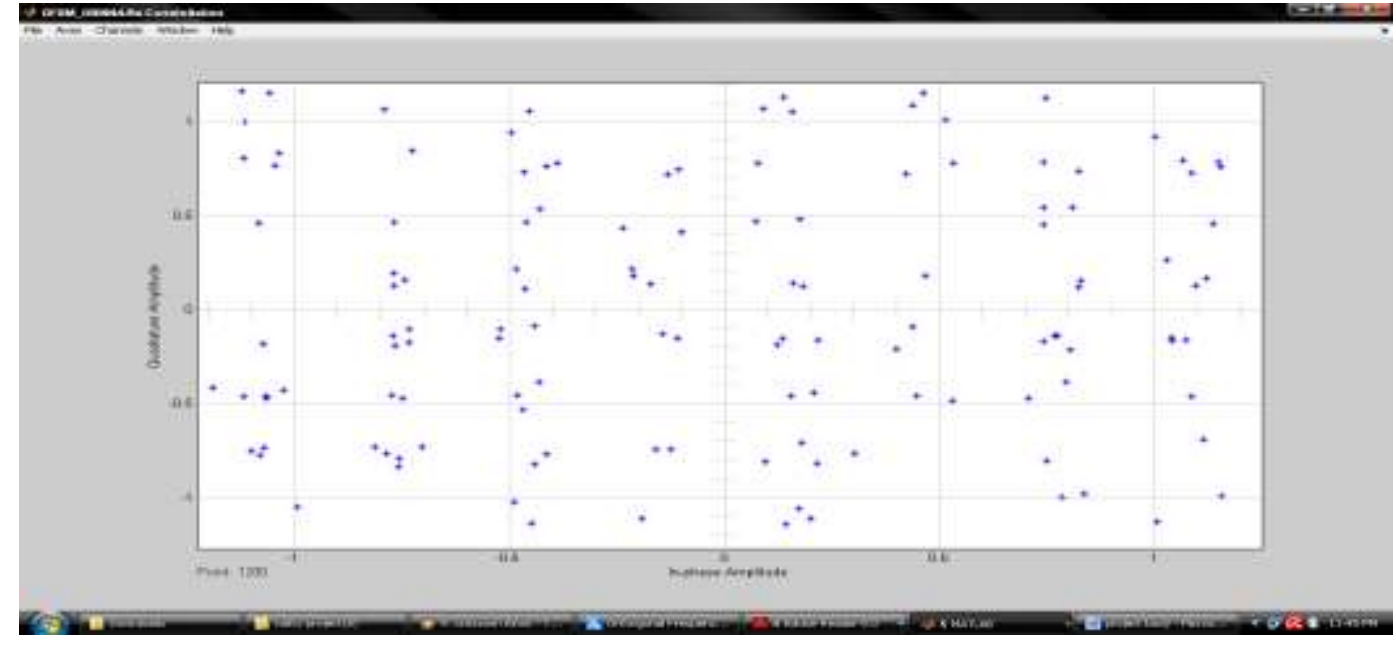

Fig 4.3 Graph of Receiver constellation 


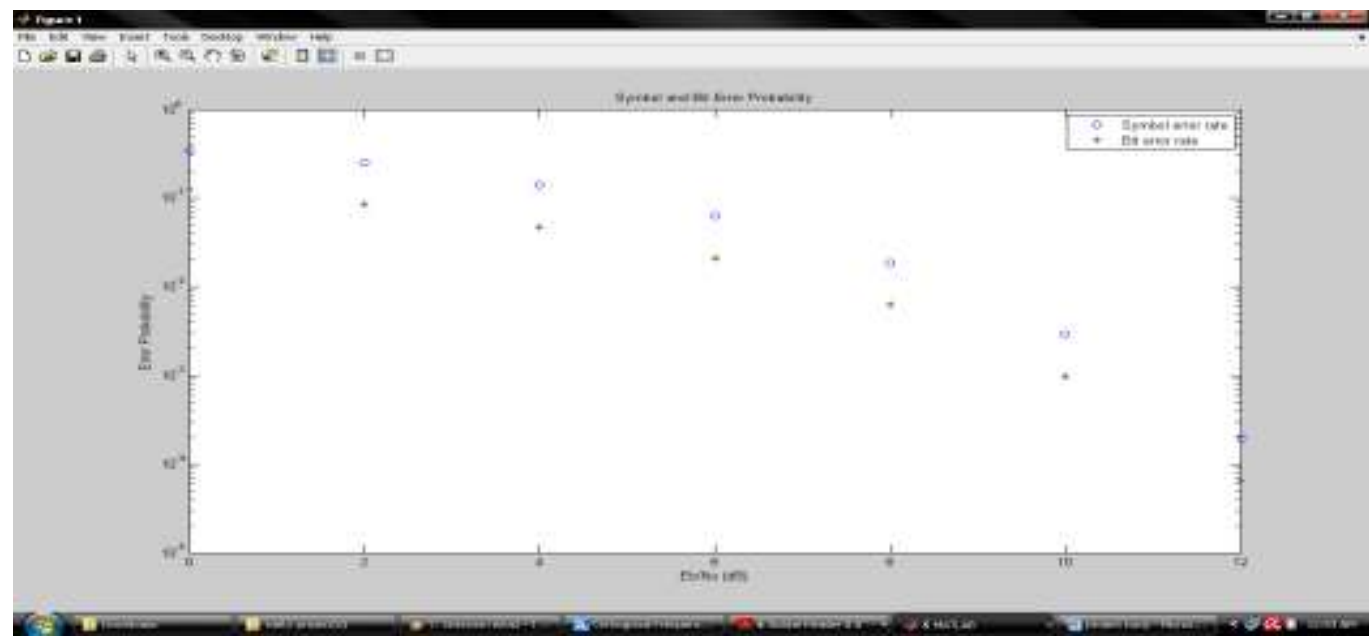

Fig 4.4 Receiver constellation

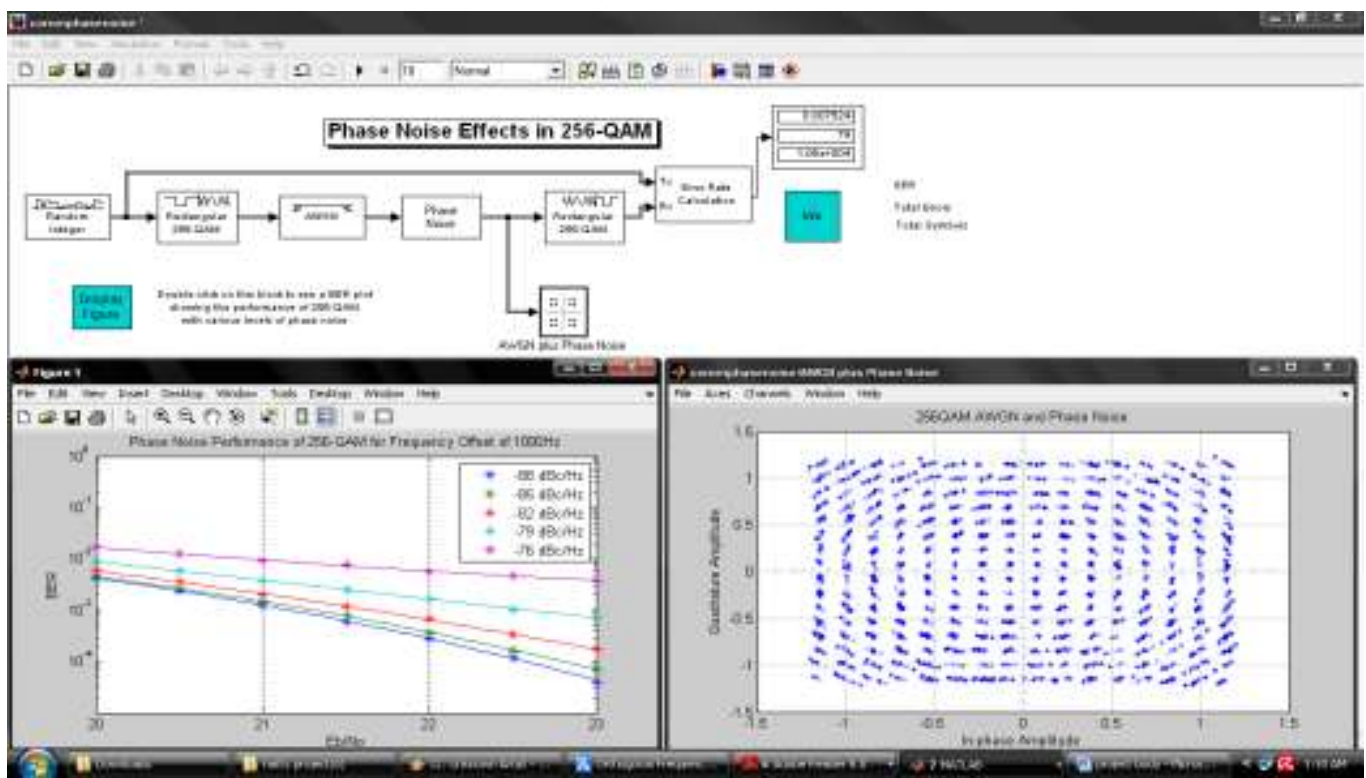

Fig 4.5 BER probability graph an OFDM modulated signal

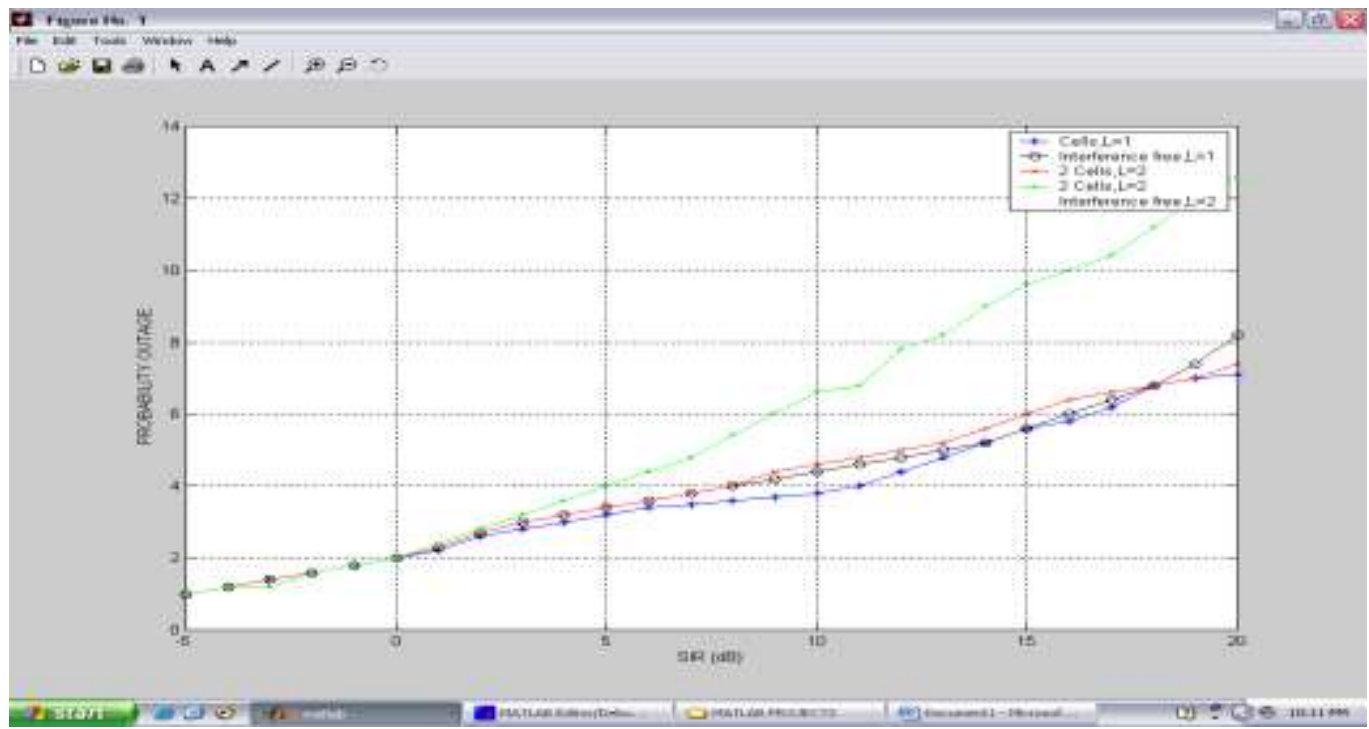

http://ijacsa.thesai.org/ 
Fig 4.6 BER Probability graph an OFDM Modulated Signal

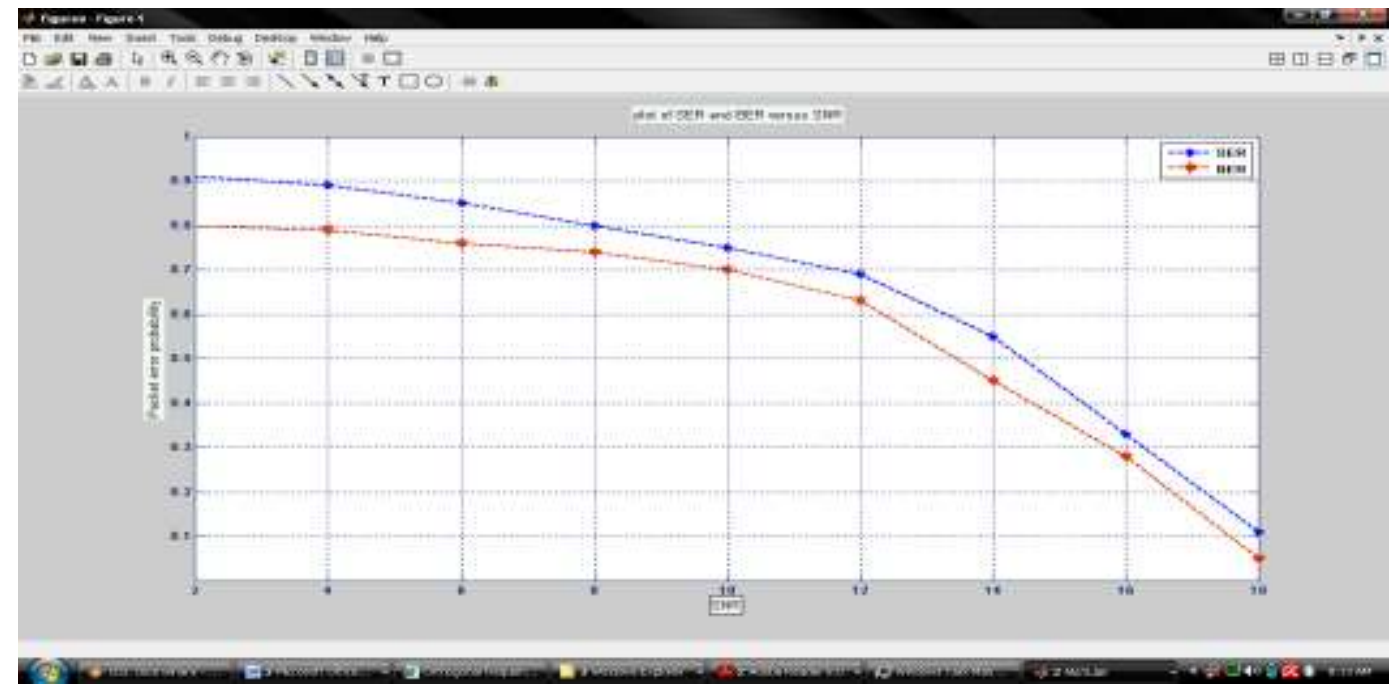

Fig 4.7 Comparison of the outage probability with the signal to interference ratio of CCI

\section{A. Deployment}

Modulation is a very important aspect of data transmission since it makes a signal more conducive for transmission over the transmission medium (in this case, the wireless channel). Hence OFDM should be widely applied to Broadband wireless access networks most especially in situations where the effects of multipath fading and noise have to be eliminated.

\section{CONCLUSION}

This work was able to show that modulation using OFDM technique is very important in Broadband wireles Access
Networks and noisy environments.The importance of modulation can be seen in the above simulations, since the unmodulated signal always performs poorer than the modulated signal. Hence, modulation makes a signal more conducive for transmission over the transmission medium (in this case, the wireless channel). This work strongly recommends OFDM as a strong candidate for Broadband Wireless Access Network.

\section{APPENDIX 1}

\section{Orthogonal Frequency Division}

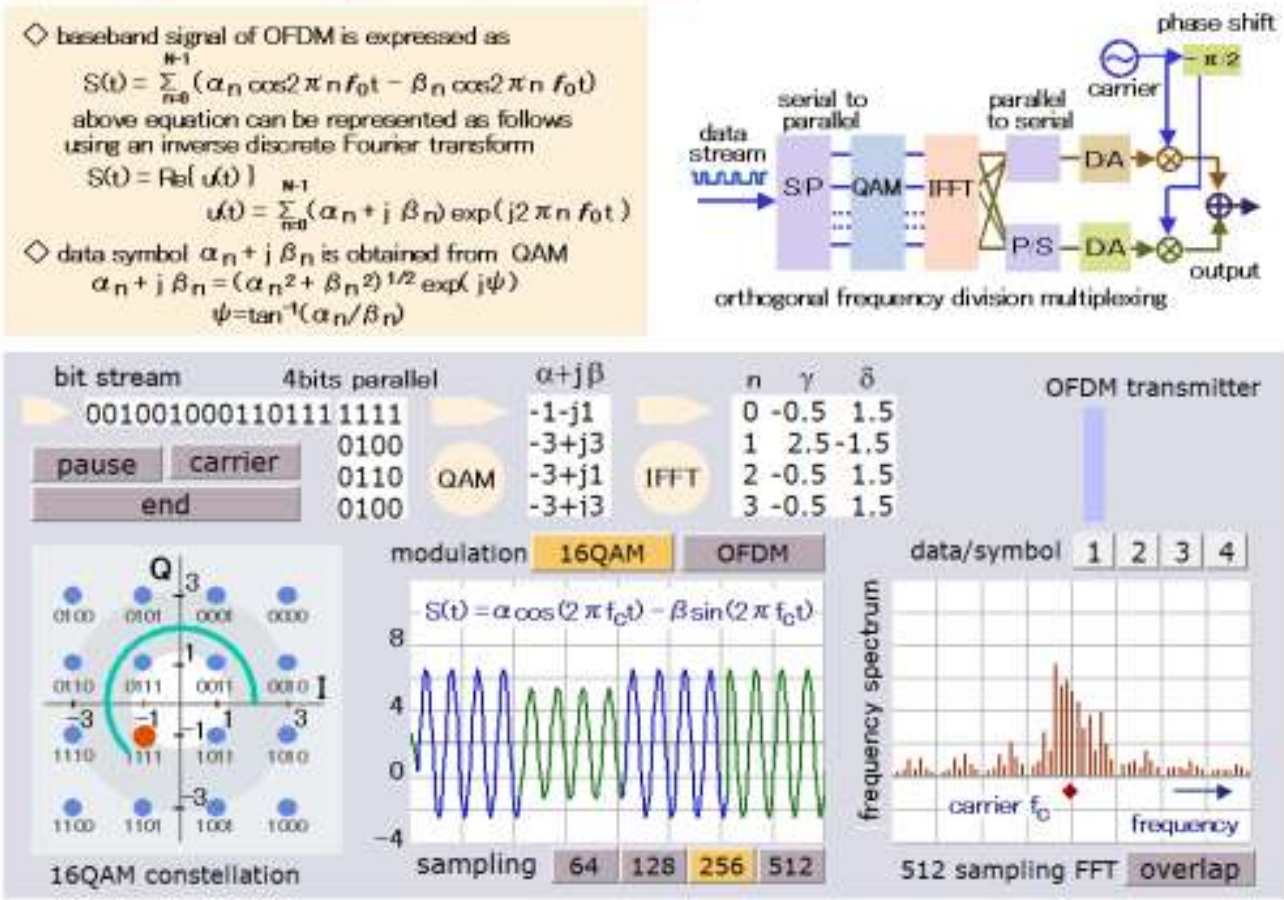

http://ijacsa.thesai.org/ 


\section{APPENDIX 2}

\section{Quadrature Phase Shift}

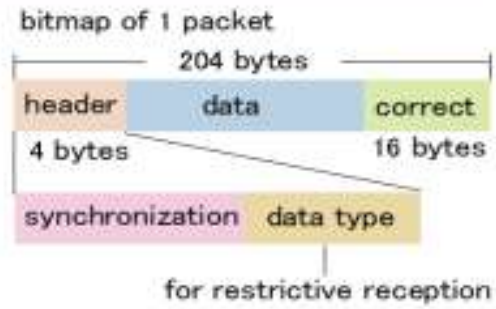

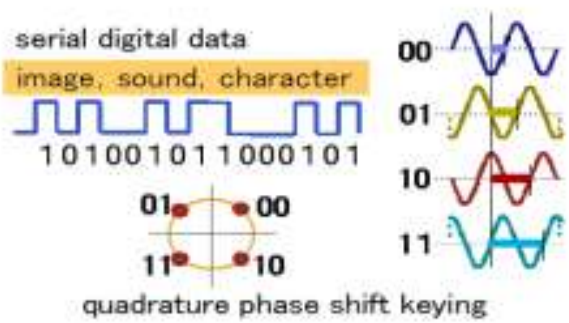

quadrature phase shift keying

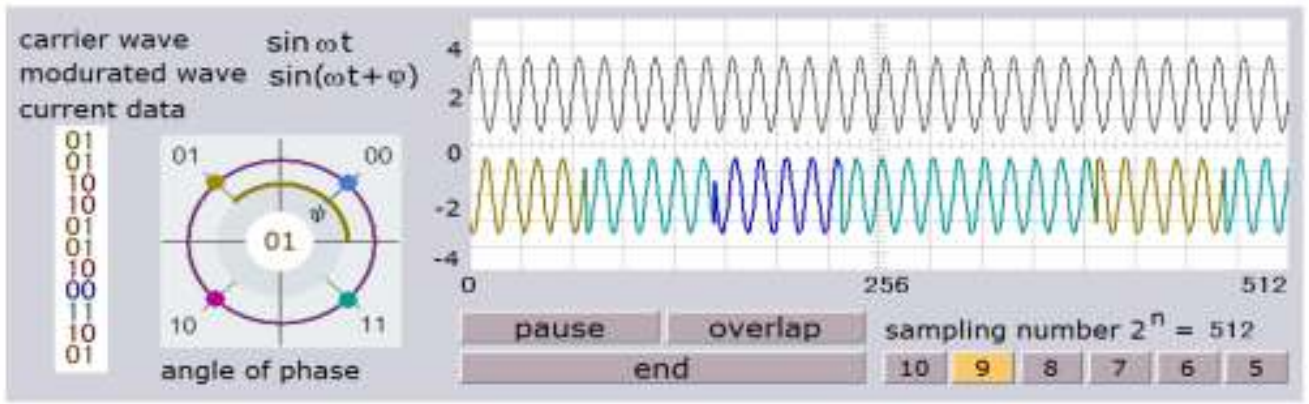

\section{REFERENCES}

[1] CIMINI, L, Analysis and simulation of digital mobile channel using OFDM. IEEE Trans. Commun., vol. 33, no. 7, pp. 665-675, 1985.

[2] Guglielmo Marconi, Early Systems of Wireless Communication: A downloadable paper (PDF) based on R.W. Simons' address to the Institution of Electrical Engineers PP112-123, 1984.

[3] S.B. Weinstein and P.M. Ebert, "Data transmission by Frequency-division multiplexing using the Discrete Fourier transform", IEEE Trans. Commun. Technol., vol. COM-19, pp. 628-634, Oct. 1971.

[4] V.E. IDIGOOrthogonal Frequency Division Multiplexing implemented as part of a Software Defined Radio (SDR) environment by Christoph Sonntag department of Electrical/Electronic Engineering University of Stellenbosch South Africa Dec. 2005

[5] V.E. IDOGHO, OFDM as a possible modulation technique for Multimedia applications in the range of $\mathrm{mm}$ waves Dušan Matiæ, Prim 10/30/98/TUD-TVS

[6] ABEL KUO, Joint Bit-Loading and Power-Allocation for OFDM,Macmillian publisher,pp1411-151, 2006

[7] E. I. Tolochko, M. Pereira and M. Faulkner, "SNR Estimation in Wireless LANs with Transmitter Diversity", in the 3rd ATcre Telecommunications and Net- working Conference, Melbourne, Australia, Dec. 2003.

[8] Fischer, Symbol-Error Rate Analysis of Fischer's Bit-Loading Algorithm, approximate analysis of the performance of Fischer's algorithm for a system with a large number of sub channels, Sept 2004.

[8] AHN, C., SASASE, I. The effects of modulation combination, target BER, Doppler frequency, and adaptive interval on the performance of adaptive OFDM in broadband mobile channel,.IEEE Trans. Consum. Electron., , vol. 48, no. 1, pp.167 - 174, Feb. 1999.

[9] YOKOMAKURA, K., SAMPEI, S., MORINAGA, N. A carrier interferometry based channel estimation technique for one-cell reuse MIMO-OFDM/TDMA cellular systems. In Proc. VTC 2006, pp. 1733$1737,2006$.

[10] YOKOMAKURA, K., SAMPEI, S., HARADA, H., MORINAGA, N. A channel estimation technique for dynamic parameter controlledOF/TDMA systems. In Proc. IEEE PIMRC, vol.1, pp. 644- 648, 2005.

[11] AHN, C. Accurate channel identification with time-frequency interferometry for OFDM. IEICE Trans. Fundamentals, vol. E90- A, no. 11, pp. 2641-2645, Nov. 2007.
[12] YOFUNE, M., AHN, C., KAMIO, T., FUJISAKA, H., HAEIWA,K. Decision direct and linear prediction based fast fading compensation for TFI-OFDM. In Proc. of ITC-CSCC2008, pp. 81 to 84, July 2008.

[13] YOSHIMURA, T., AHN, C., KAMIO, T., FUJISAKA,H.,HAEIWA, K. Performance enhancement

\section{AUTHORS PROFILE}

Engr. James Agajo is into a Ph.D Programme in the field of Electronic and Computer Engineering, He has a Master's Degree in Electronic and telecommunication Engineering from Nnamdi Azikiwe University Awka Anambra State, and also possesses a Bachelor degree in Electronics and Computer Engineering from the Federal University of Technology Minna Nigeria. His interest is in intelligent system development with a high flare for Engineering and Scientific research. He has Designed and implemented the most resent computer controlled robotic arm with a working grip mechanism 2006 which was aired on a national television, he has carried out work on using blue tooth technology to communicate with microcontroller. Has also worked on thumb print technology to develop high tech security systems with many more $\mathrm{He}$ is presently on secondment with UNESCO TVE as a supervisor and a resource person. James is presently a member of the following association with the Nigeria Society of Engineers(NSE), International Association of Engineers(IAENG) UK, REAGON, MIRDA,MIJICT.

Dr. Isaac Avazi Omeiza holds B.Eng, M.Eng and Ph.D degrees in Electrical/Electronics Engineering. His lecturing career at the university level has spanned a period of about two decades. He has lectured at the Nigerian Defence Academy Kaduna( the Nigerian Military university), University of Ilorin and the 'Capital-City-University of Nigeria' - University of Abuja. He has also been a member of the Nigerian society of Engineers ( NSE) and a member of the Institute of Electrical and Electronic Engineers of America ( IEEE ). He has supervised several undergraduate final-year projects in Electronic designs and has done a number of research works in digital image processing, Fingerprint processing and the processing of video signals.

Engr. Joseph Okhaifoh is into a PH.D programme, he holds a Master's degree in Electronics and telecommunication Engineering and a Bachelor Degree in Electrical and Electronics Engineering, he is presently a member of Nigeria society of Engineers.

Dr V.E Idigo holds a Ph.D, M.Eng, BEng. in Communication Engineering, a Member of IAENG,MNSE and COREN, he is presently the Head of Department of Electrical Electronics in Nnamdi Azikiwe University Awka Anambra State, Nigeria 